\title{
Pattern Formation in Low-Pressure Radio-Frequency Plasmas due to a Transport Instability
}

\author{
Victor Désangles, ${ }^{1,2}$ Jean-Luc Raimbault, ${ }^{2}$ Alexandre Poyé, ${ }^{1,3}$ Pascal Chabert, ${ }^{2}$ and Nicolas Plihon $\oplus^{1}$ \\ ${ }^{1}$ Univ Lyon, Ens de Lyon, Univ Claude Bernard, CNRS, Laboratoire de Physique, F-69342 Lyon, France \\ ${ }^{2}$ Laboratoire de Physique des plasmas, CNRS, Sorbonne Université, Université Paris Sud, École Polytechnique, \\ F-91120 Palaiseau CEDEX, France \\ ${ }^{3}$ Aix-Marseille Université, CNRS, PIIM, UMR 7345, Marseille, France
}

(Received 1 July 2019; revised manuscript received 9 October 2019; published 23 December 2019)

Pattern formation, observed experimentally in a radio-frequency plasma in annular geometry, and characterized by azimuthal symmetry breaking of the plasma parameters, is reported. The azimuthal modulation increases with increasing pressure in the range 1-300 Pa. These experimental observations are accurately described by a fluid model in which the transport coefficients are computed from a OD Boltzmann kinetic equation. A linear stability analysis shows that unstable modulations develop at low and intermediate pressures, following an instability mechanism due to an energy transport effect-the instability mechanism lies in the sign of off-diagonal terms for the electron particles and energy fluxes expressed as functions of gradients of the plasma density and the electron temperature. This model is an excellent candidate to explain the occurrence of striations in radio-frequency plasmas.

DOI: 10.1103/PhysRevLett.123.265001

Introduction.-Pattern formation is a ubiquitous phenomenon in plasmas, arising from interaction with boundaries [1], volumetric processes, or spatiotemporal complexity [2]. Striations are a particular form of self-organization observed in various conditions: dc discharges [3], magnetic mirrors [4], laser generated plasmas [5], and ionospheric plasmas $[6,7]$. In dc nonequilibrium gas discharge plasmas driven at intermediate neutral pressures (10-1500 Pa), striations are usually interpreted as an ionization instability. The source of this specific instability is generally recognized in the fact that the ionization frequency is a function of the electron density, a situation that arises when stepwise ionization of excited atoms is important $[3,8,9]$. Striations have also been observed in radio-frequency (rf) discharge plasmas [10-12], but the physical origin of this phenomena is not as well understood, despite numerical investigations [13]. This is particularly clear in the low-pressure regimes where direct ionization is predominant against metastable two-step ionization which cannot be considered at the origin of the instability [14-16]. In this Letter, we propose a model where the ionization instability leading to striations is not driven by stepwise ionization but mainly by a transport process which affects the electron energy flow and tends to reinforce the electron temperature fluctuations. Computing the transport coefficients from a 0D Boltzmann equation, we extend a previous model [17], showing good agreement with a detailed experimental characterization of the formation of striations in an annular if plasma.

Striations formation in a rf plasma.-The experimental setup, sketched in Fig. 1(a), consists of a cylindrical pyrex tube of radius $R_{2}=64 \mathrm{~mm}$ and height $H=118 \mathrm{~mm}$ terminated by two metal flanges. A smaller pyrex tube of radius $R_{1}=16 \mathrm{~mm}$ is inserted through the top flange and hosts a 14-turns coil. The plasma is created by a $5.8 \mathrm{MHz}$ rf current circulating in the coil. The coil is divided in three sections (5-4-5 turns) connected in series with capacitors in order to minimize the coil rf potential
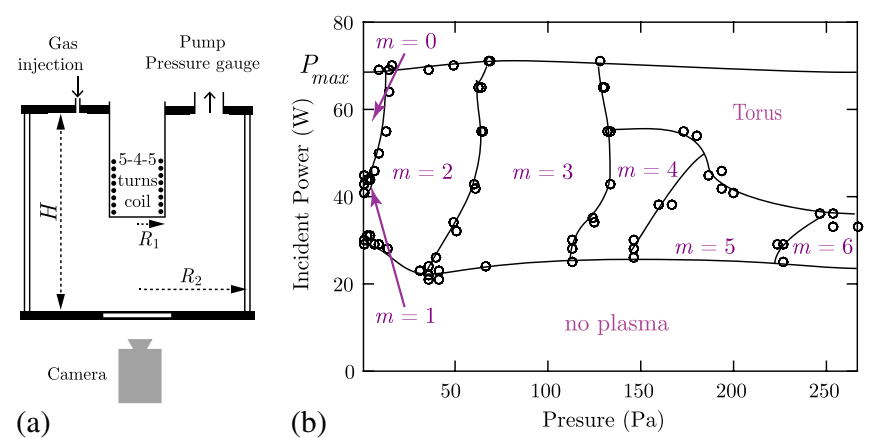

(a)

(b)

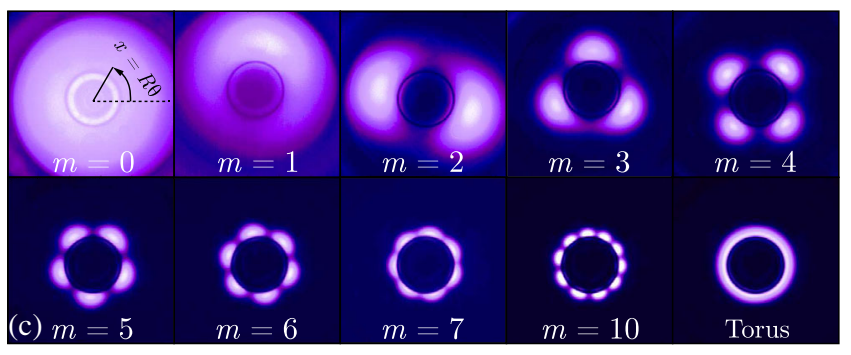

FIG. 1. (a) Experimental setup. (b) Observed azimuthal modulation mode $m$ in incident power vs pressure parameter space. (c) Typical plasma optical emission recorded by the camera. See text for details. 
with respect to ground [18]. As a consequence, this design cannot operate in the capacitive regime and the plasma may only be sustained from inductive coupling (requiring a minimum power of typically $20 \mathrm{~W}$ ). Argon gas is injected at constant flow rate and a valve at the entrance of the pump controls the operating neutral pressure, ranging from 1 to $300 \mathrm{~Pa}$. The pressure is measured using a combined cold cathode-pirani Pfeiffer PKR251 gauge. A Bird 43 watt meter measured the incident and reflected powers delivered to the rf matchbox. The vertical integrated plasma optical emission is observed from the opposite flange, fitted with a $50 \mathrm{~mm}$ diameter optical window using a IO-Flare 2MP360 camera $(1080 \times 1080$ pixels, 8 bits gray scale images, at a rate of $360 \mathrm{fps})$. Experiments reported in this Letter involve plasma densities of a few $10^{10}$ to a few $10^{11} \mathrm{~cm}^{-3}$ [18].

The striations reported in this Letter consist of pattern formation breaking the azimuthal symmetry of the setup. As a consequence, the optical emission as recorded from the camera and displayed in Fig. 1(c), shows modulations of the form $\cos (m \theta)$, where $\theta$ is the azimuthal angle and $m$ an integer. Let us first describe the top-left panel which shows an azimuthally homogeneous plasma observed at the lowest pressures and highest powers (the internal tube is observed brighter, and the plasma diffuses downstream of the internal tube). In this regime, the azimuthal symmetry is preserved, corresponding to an absence of azimuthal modulation (i.e., $m=0$ ). This homogeneous regime is labeled 0 (according to the value of the modulation mode number $m$ ) in the parameter space (pressure, incident power) displayed in Fig. 1(b) and is observed below $10 \mathrm{~Pa}$ and above $40 \mathrm{~W}$. As the pressure is gradually increased, the plasma parameters show azimuthal modulations with increasing values of $m$. The panels of Fig. 1(c) from left to right show typical observations of these regimes with increasing values of $m$. The light intensity recorded by the camera is integrated over the full visible spectrum and the synthetic color map used in Fig. 1(c) was chosen to mimic the visible spectrum. The experimental points displayed as open circles in Fig. 1(b) correspond to transitions between regimes observed as the pressure was increased, at constant incident power delivered to the antenna. We stress here that bistability between adjacent modulated regimes was observed. For instance, the transition from the $m=0$ azimuthally symmetric regime to the $m=2$ regime was observed to occur at $13 \mathrm{~Pa}$ (i.e., increasing the pressure), while the transition from $m=2$ to the $m=0$ regime was observed at $3 \mathrm{~Pa}$ (i.e., decreasing the pressure) - for $56 \mathrm{~W}$ incident power. The boundaries reported in Fig. 1(b) should thus be regarded as indicative when compared to the linear instability analysis presented below. Moreover, the modulated regimes were also observed to slowly rotate (at a few $\mathrm{rad} \mathrm{s}^{-1}$ ), with rotation rates highly sensitive to details of the experimental setup (such as neutral gas or vessel temperature drifts, presence of intrusive probes). These features will not be discussed further in this Letter. For the highest pressures and high incident power, the plasma recovers the azimuthal symmetry: a torus-shaped plasma is observed around the internal tube [see bottom right panel of Fig. 1(c)]. A linear instability model is derived in the remainder of this Letter, which reproduces the main experimental observations: (i) the modulation wave number increases with increasing neutral pressure and (ii) axisymmetry is recovered at large pressures.

Model for the striation formation.-We use a modified version of an electron fluid model proposed initially by Ingold for the modeling of the nonequilibrium dc positive column [19]. The validity of a fluid model is ensured at the lowest investigated pressure $(1 \mathrm{~Pa})$, for which the ion-mean free path is of the order of $3 \mathrm{~mm}$, i.e., lower than the radial extension $\left(R_{2}-R_{1}\right)$. This model-sometimes called a nonlocal moment model-differs from the usual local moment in two main aspects. First, the balance equations are derived by taking moments of the Boltzmann equation after the two-term expansion of the energy distribution function is made. Second, the transport and rate coefficients appearing in the electron fluid equations are not calculated from the usual Maxwellian assumption but are parametrized by the average electron energy (or electron temperature) by the numerical solutions of the 0D Boltzmann equation for different values of the electric field. Although it is generally believed that a kinetic approach is necessary for a precise modeling of discharges at low pressure, it was shown by Ingold that the fluid nonlocal moment model shows good agreement with the results obtained by the numerical solutions of the 1D kinetic Boltzmann equation.

For the sake of simplicity, and to extract the physical mechanisms at work, we ignore the axial dependence of all plasma parameters. Introducing a linear coordinate $x=R_{m} \theta$, where $R_{m}=2.8 \mathrm{~cm}$ is the radius of the maximum plasma density $[18,20]$ and neglecting curvature, the particle and energy balance equations for the radially integrated electron density $n_{e}$ and electron temperature $T_{e}$ read

$$
\begin{gathered}
\partial_{t} n_{e}+\partial_{x} \Gamma_{e}=\left(\nu_{i z}\left(T_{e}\right)-\nu_{r}\right) n_{e} \\
\partial_{t}\left(3 / 2 n_{e} T_{e}\right)+\partial_{x} H_{e}=P_{0} n_{e}^{B}-n_{e} \nu_{i z}\left(T_{e}\right) \mathcal{E}_{c}\left(T_{e}\right) .
\end{gathered}
$$

Here, $\nu_{i z}\left(T_{e}\right)$ is the ionization frequency, $\nu_{r}$ accounts for the radial losses of particles at $R_{1}$ and $R_{2}, P_{0}$ is the normalized power density, $\mathcal{E}_{c}$ is the collisional energy loss per electron-ion pair created [21] and $B$ depends on the type of electron heating mechanisms. Here, we will restrict our study to the case of pure inductive coupling, i.e., $B=-1 / 2$-however it is easily shown that this choice only slightly modifies the dispersion relation and the values of the most unstable modes but does not modify the conclusions of our investigation. 
Using the two-term approximation for the electron distribution function, the electron particles and energy fluxes $\Gamma_{e}$ and $H_{e}$ in Eqs. (1)-(2) take the form

$$
\begin{gathered}
\Gamma_{e}=-n_{e} \mu_{e} E-\partial_{x}\left(n_{e} D_{e}\right), \\
H_{e}=-n_{e} \beta_{e} E-\partial_{x}\left(n_{e} G_{e}\right) .
\end{gathered}
$$

Here $D_{e}$ and $\mu_{e}$ denote the well-known coefficients of electron diffusion and mobility, respectively, while $G_{e}$ and $\beta_{e}$ are the electron energy diffusion and electron energy mobility coefficients [22]. For a quasineutral plasma within the ambipolar assumption, $\Gamma_{e}=\Gamma_{i}$, when the ion motion is mobility limited, the electric field along the azimuthal direction $E$ is given by the relation $E=\Gamma_{e} /\left(n_{e} \mu_{i}\right)$, where $\mu_{i}$ is the ion mobility. Hence, the electric field can be eliminated from Eqs. (3)-(4) leading to the following expressions for $\Gamma_{e}$ and $H_{e}$ :

$$
\begin{gathered}
\Gamma_{e}=-D_{a} \partial_{x} n_{e}-\eta_{e} \partial_{x} T_{e} \\
H_{e}=-\chi_{e} \partial_{x} n_{e}-\kappa_{e} \partial_{x} T_{e} .
\end{gathered}
$$

In these equations, $D_{a}=\mu_{i} D_{e} / \mu_{e}$ is the ambipolar diffusion (we used the inequality $\mu_{i} / \mu_{e} \ll 1$ ), $\kappa_{e}=n_{e}\left[\partial_{T_{e}} G_{e}-\left(\beta_{e} / \mu_{e}\right) \partial_{T_{e}} D_{e}\right]$ is the usual electron thermal conductivity while the off-diagonal terms, $\eta_{e}=n_{e}\left(\mu_{i} / \mu_{e}\right) \partial_{T_{e}} D_{e}$ and $\chi_{e}=G_{e}-\left(\beta_{e} / \mu_{e}\right) D_{e}$ lead to fluxes similar to the Soret effect (or thermophoresis) and the Dufour effect [23]. These coefficients can be obtained from the 0D Boltzmann code Bolsig+ [24] as functions of the electron temperature, defined as $2 / 3$ times the electron mean energy [25]. The values of the ionization frequency $\nu_{i z}\left(T_{e}\right)$ and the total power losses $\nu_{i z}\left(T_{e}\right) \mathcal{E}_{c}\left(T_{e}\right)$, taking into account elastic and inelastic processes, are also computed using the Bolsig+ code.

Note that it can be shown that $\chi_{e}=0$ and $\eta_{e}>0$ when the electron function distribution is Maxwellian and the collision for momentum transfer is assumed independent of the electron velocity [22]. From the Bolsig+ simulations, $\chi_{e}$ and $\eta_{e}$ appear to be strictly negatives for argon. Consequently, contrary to the diagonal terms, $-D_{a} \partial_{x} n_{e}$ and $-\kappa_{e} \partial_{x} T_{e}$ which tend to smooth out any temperature or density gradients, the off-diagonal terms, $-\eta_{e} \partial_{x} T_{e}$ and $-\chi_{e} \partial_{x} n_{e}$ tend to reinforce the electron density or electron temperature fluctuations. As shown in the following, an instability can occur when these destabilizing contributions dominate.

Equilibrium plasma parameters.-The stationary and homogeneous equilibrium electron density $n_{0}$ and electron temperature $T_{0}$ are given from Eqs. (1) and (2) by the implicit solutions of the equations $\nu_{r}=\nu_{i z}\left(T_{0}\right)$ and $P_{0} n_{0}^{B}=n_{0} \nu_{r} \mathcal{E}_{c}\left(T_{0}\right)$. The instability criterion derived below is independent of $n_{0}$, one thus focuses on the value of $T_{0}$. Assuming a constant and uniform value for $T_{0}$ in the

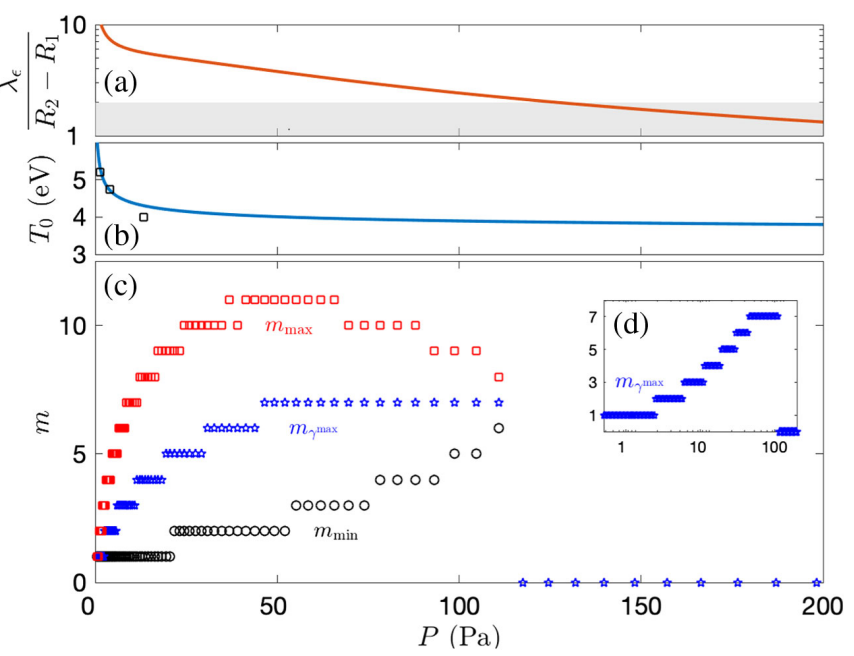

FIG. 2. (a) Normalized energy relaxation length $\lambda_{\epsilon} /\left(R_{2}-R_{1}\right)$, (b) electron temperature $T_{0}$ computed from the annular equilibrium model, (c) unstable azimuthal mode numbers $m$ as a function of pressure in linear scale, and (d) logarithmic scale. See text for details.

annular section, the radial profile of the electron density $n(r)$ is obtained from the diffusion equation

$$
\frac{1}{r} \frac{d}{d r}\left(r \frac{d n}{d r}\right)+\beta^{2} n(r)=0,
$$

with $\beta^{2}=\nu_{i z}\left(T_{0}\right) / D_{a}\left(T_{0}\right)$. The boundary conditions impose the out fluxes $-D_{a} d n /\left.d r\right|_{R_{1,2}}= \pm n\left(R_{1,2}\right) u_{B}$, where $u_{B}=\left(T_{0} / M_{i}\right)^{1 / 2}$ is the Bohm velocity with $M_{i}$ the ion mass. Introducing $\psi=u_{B} / D_{a}, T_{0}$ is computed from the following equation [26]:

$$
\begin{aligned}
& \left(\beta J_{1}\left(\beta R_{1}\right)+\psi J_{0}\left(\beta R_{1}\right)\right)\left(\beta Y_{1}\left(\beta R_{2}\right)-\psi Y_{0}\left(\beta R_{2}\right)\right) \\
& =\left(\beta Y_{1}\left(\beta R_{1}\right)+\psi Y_{0}\left(\beta R_{1}\right)\right)\left(\beta J_{1}\left(\beta R_{2}\right)-\psi J_{0}\left(\beta R_{2}\right)\right),
\end{aligned}
$$

where $J_{0}$ and $Y_{0}$ (respectively, $J_{1}$ and $Y_{1}$ ) are zero order (respectively, first order) Bessel functions of the first and second kind. The solid line in Fig. 2(b) shows the evolution of the equilibrium temperature $T_{0}$ as a function of pressure, computed from Eq. (7). The ionization frequency is computed using the Bolsig+ code, assuming a temperature of $500 \mathrm{~K}$ for the neutral gas, and the ion mobility is computed in the collision dominated limit as $\mu_{i}=e /\left(M_{i} N_{g} \sigma_{i} v_{\text {thi }}\right)$ with $v_{\text {thi }}$ the ion thermal velocity, $\sigma_{i}=1.74 \times 10^{-18} \mathrm{~m}^{2}$ the cross section for ion-neutral collisions at $700 \mathrm{~K}$ [27]. A good agreement is observed with the values of the electron temperature reported from PIC simulations in the same geometry [20], estimated at $r=R_{m}$, and reported as symbols in Fig. 2(b).

Linear stability analysis. - To investigate the stability of the plasma around the equilibrium state $\left(n_{0}, T_{0}\right)$, we introduce small density and temperature perturbations $\delta n_{e}$ and 
$\delta T_{e}$ such that $n_{e}=n_{0}+\operatorname{Re}\left(\delta n_{e} e^{\gamma t+i k x}\right), \quad T_{e}=T_{0}+$ $\operatorname{Re}\left(\delta T_{e} e^{\gamma t+i k x}\right)$, where $\gamma$ is the growth rate in time and $k$ is the perturbation wave number. Substituting these expressions in the continuity Eq. (1) and using Eq. (5), we obtain the dispersion relation

$$
\gamma=-D_{a}^{0} k^{2}+\left.n_{0} \frac{\partial \nu_{i z}}{\partial T}\right|_{T_{0}} \frac{\delta T_{e}}{\delta n_{e}},
$$

where $\mathcal{Z}^{0}$ denotes a parameter $\mathcal{Z}$ evaluated at $T_{e}=T_{0}$, and we have neglected $\eta_{e} \partial_{x} T_{e}$ in Eq. (5). Thus, the dispersion relation has two contributions: one due to diffusion and one coming from the fluctuations of electron temperature induced by the density fluctuations. Clearly, since the diffusion contribution is always negative, a destabilizing effect occurs only if $\delta T_{e} / \delta n_{e}$ is positive enough. The evaluation of $\delta T_{e} / \delta n_{e}$ is obtained after the linearization of Eq. (2), using Eq. (6) and neglecting the small term $\partial_{t}\left(3 / 2 n_{e} T_{e}\right)$ :

$$
\frac{\delta T_{e}}{\delta n_{e}}=-\frac{3 \mathcal{E}_{c}^{0} \nu_{i z}^{0} / 2+k^{2} \chi_{e}^{0}}{\left.n_{0}\left[\partial\left(\nu_{i z} \mathcal{E}_{c}\right) / \partial T\right]\right|_{T_{0}}+k^{2} \kappa_{e}^{0}} .
$$

This result shows that $\delta T_{e} / \delta n_{e}$ is positive if the transport coefficient $\chi_{e}^{0}$ is itself negative enough. Since $\nu_{i z}^{0} \propto N_{g}$ and $\chi_{e}^{0} \propto N_{g}^{-1}$, a destabilizing effect is helped at low pressures. Combining Eq. (8) and Eq. (9), a condition for the instability can be expressed as $\mathcal{A} k^{4}+\mathcal{B} k^{2}+\mathcal{C}<0$, where $\mathcal{A}, \mathcal{B}$, and $\mathcal{C}$ depend on the parameters previously introduced. It can be checked that a necessary and sufficient condition for this inequality is

$$
\begin{aligned}
\chi_{e}^{0}< & \left(\left.\frac{\partial \nu_{i z}}{\partial T}\right|_{T_{0}}\right)^{-1}\left(-\left.D_{a}^{0} \frac{\partial\left(\nu_{i z} \mathcal{E}_{c}\right)}{\partial T}\right|_{T_{0}}\right. \\
& -\sqrt{\left.\left.\frac{6 D_{a}^{0} \kappa_{e}^{0}}{n_{0}} \mathcal{E}_{c}^{0} \nu_{i z}^{0} \frac{\partial \nu_{i z}}{\partial T}\right|_{T_{0}}\right) .}
\end{aligned}
$$

This implies that the transport coefficient $\chi_{e}^{0}$ must be sufficiently negative in order to induce the instability of the homogeneous and steady state $\left(n_{0}, T_{0}\right)$. Note that this condition is independent of the value of the equilibrium plasma density $n_{0}$ since $\kappa_{e}^{0} / n_{0}$ is independent of $n_{0}$.

Discussion and comparison with experimental results.When the instability condition is met, the fourth order polynomial in $k$ is negative in a range of $k$ values extending from $k_{\min }$ to $k_{\max }$ and the wave vector $k_{\gamma^{\max }}$ of the most unstable perturbation corresponds to the most positive value of $\gamma$. As the transport coefficients strongly depend on the equilibrium electron temperature $T_{0}$, the details of the instability described above strongly depend on the neutral pressure. Let us now investigate the influence of neutral pressure on the unstable modes, and show that the instability indeed reproduces the experimental features displayed in Fig. 1(b). Axisymmetry of the experimental configuration imposes periodic boundary conditions on the perturbed quantities [i.e., $\delta n_{e}(x=0)=\delta n_{e}(x=L)$ ]; wave numbers $k$ are thus restricted to $k=2 \pi m$, where $m$ is the azimuthal mode number. Figure 2(c) shows the evolution of the unstable azimuthal mode $m$ when solving the dispersion relation given by Eqs. (8) and (9). Unstable modes are obtained for $m_{\min }<m<m_{\max }$ and the most unstable mode $m_{\gamma^{\max }}$ lies within this range. A first observation is that our model reproduces the main feature of the experimental configuration: as the pressure increases, the most unstable mode number $m_{\gamma^{\max }}$ increases with a range of azimuthal modulations similar to that of the experiment. A second observation is that the system is linearly stable above $115 \mathrm{~Pa}$ and thus axisymmetry is recovered at high pressures - the most unstable wave number reported in Fig. 2(c) being null. We emphasize here that the model is restricted to the linear phase of the instability, while the experimentally observed mode is the nonlinearly saturated regime. Since bistability exists in the experiment, we do not expect the model to exactly predict the values for the observed transitions between modes as presented in Fig. 1(b). Closer investigation of the dispersion relation (not shown) shows that, for a constant value of $T_{0}$, the most unstable mode increases with increasing pressure, while, for a constant neutral pressure, the most unstable mode decreases with decreasing $T_{0}$. The simplifying hypothesis of our model, which considers a uniform electron temperature in the annular section, seems to lead to overestimated electron temperature values as compared to those reported in PIC simulations [20]. In turn, this overestimates the value of the most unstable wave number. A first improvement would thus to incorporate a transport equation for the energy, especially for regimes when the energy relaxation length $\lambda_{\epsilon}$ [28] is not significantly larger than the typical radial extension of the configuration. Figure 2(a) displays $\lambda_{\epsilon} /\left(R_{2}-R_{1}\right)$ and shows that for pressure above $130 \mathrm{~Pa}$, the energy relaxation length is lower than twice the radial size of the experiment. A detailed computation of the equilibrium electron temperature for pressures above typically $100 \mathrm{~Pa}$ would thus require a transport equation for the energy - a task beyond the scope of the present Letter. We note from Fig. 1(c) that, indeed at large pressures, the radial extension of the plasma is restricted to locations close to the inner tube and that this strongly inhomogeneous situation may only be captured when adding an energy transport equation.

Conclusion.-In this Letter, we developed an electron fluid model in which the transport coefficients are computed from a OD Boltzmann equation. A linear stability analysis shows that striations may develop at low and intermediate pressures, following an instability mechanism due to a transport effect, in contrast with the current understanding of the striations in dc positive columns. 
The instability mechanism lies in the sign of off-diagonal terms for the electron particles and energy fluxes expressed as functions of gradients of the plasma density and the electron temperature. This model reproduces the features of unstable modes experimentally observed in an inductive radio-frequency created plasma in an annular vacuum vessel. The model reported in the present Letter thus proposes a new framework to understand pattern formation observed in radio-frequency plasma discharges. A first improvement of this work would be a detailed study of the nonlinear saturation mechanisms of this instability. A systematic investigation of the influence of the nature of the gas on the development of the instability should also be carried out experimentally and numerically, with an emphasis on atomic and molecular gases.

The authors are thankful to Miles Turner for fruitful discussions and to Valery Godyak for the design of the experiment. N. P., V. D., and A. P. acknowledge support by the French National Research Agency under Contract No. ANR-13-JS04-0003-01.

[1] J. P. Trelles, J. Phys. D 49, 393002 (2016).

[2] P. H. Diamond, S.-I. Itoh, and K. Itoh, Modern Plasma Physics, Vol. 1 (Cambridge University Press, Cambridge, England, 2010).

[3] V. I. Kolobov, J. Phys. D 39, R487 (2006).

[4] A. J. Balloni, S. Aihara, and P. H. Sakanaka, Plasma Phys. Controlled Fusion 30, 1659 (1988).

[5] Y. Hoshi, H. Yoshida, and Y. Tsutsui, J. Appl. Phys. 92, 5668 (2002).

[6] L. M. Linson and J. B. Workman, J. Geophys. Res. 75, 3211 (1970).

[7] A. V. Gurevich, K. P. Zybin, and A. V. Lukyanov, Phys. Rev. Lett. 75, 2622 (1995).

[8] Y.P. Raizer, Gas Discharge Physics (Springer-Verlag, Berlin, 1991).

[9] B. M. Smirnov, Physics of Ionized Gases (John Wiley \& Sons, Inc., New York, 2001).
[10] M. Chenot, Recherches expérimentales sur la décharge en haute fréquence, $\mathrm{Ph}$. D. thesis, Faculté des Sciences de l'Université de Paris, 1947.

[11] A. S. Penfold, J. A. Thornton, and R. C. Warder, Czech. J. Phys. B 23, 431 (1973).

[12] Y.-X. Liu, E. Schüngel, I. Korolov, Z. Donkó, Y.-N. Wang, and J. Schulze, Phys. Rev. Lett. 116, 255002 (2016).

[13] K. Denpoh, Jpn. J. Appl. Phys. 51, 106202 (2012).

[14] C. M. Ferreira, J. Loureiro, and A. Ricard, J. Appl. Phys. 57, 82 (1985).

[15] D. P. Lymberopoulos and D. J. Economou, J. Appl. Phys. 73, 3668 (1993).

[16] Y.-K. Lee and C.-W. Chung, J. Appl. Phys. 109, 013306 (2011).

[17] D. Mackey, L. Plantié, and M. Turner, Appl. Math. Lett. 18, 865 (2005).

[18] J. Arancibia Monreal, P. Chabert, and V. Godyak, Phys. Plasmas 20, 103504 (2013).

[19] J. H. Ingold, Phys. Rev. E 56, 5932 (1997).

[20] S.-H. Song, Y. Yang, P. Chabert, and M. J. Kushner, Phys. Plasmas 21, 093512 (2014).

[21] M. A. Lieberman and A. J. Lichtenberg, Principles of Plasma Discharges and Materials Processing, 2nd ed. (John Wiley \& Sons, Inc., New York, 2005).

[22] W. P. Allis and S. J. Buchsbaum, in Selected Works of William Phelps Allis, edited by S. Brown (MIT Press, Cambridge, MA, 1967), pp. 171-173.

[23] L. S. García-Colín, A. L. García-Perciante, and A. SandovalVillalbazo, Phys. Plasmas 14, 012305 (2007).

[24] G. J. M. Hagelaar and L. C. Pitchford, Plasma Sources Sci. Technol. 14, 722 (2005).

[25] The azimuthal rf heating field does not induce any anisotropy in the plasma on a timescale characteristic of the development of the instability; the values of the transport coefficients in the radial and in the azimuthal directions are thus identical and given by the transverse Bolsig+ transport coefficients.

[26] Y. Zhang, C. Charles, and R. Boswell, Phys. Plasmas 22, 073510 (2015).

[27] A. V. Phelps, J. Appl. Phys. 76, 747 (1994).

[28] P. Chabert and N. Braithwaite, in Physics of RadioFrequency Plasmas (Cambridge University Press, Cambridge, England, 2011), p. 394. 